\title{
Smoothness of Connection of Ruled Road Surface Form Segments
}

\author{
Evgeniy Lyubchinov ${ }^{1}$ and Konstantin Panchuk ${ }^{1}$ \\ ${ }^{1}$ Omsk State Technical University, Mira, h. 11, Omsk, 644050, Russia
}

\begin{abstract}
Since the modern systems of automated road surface form design have allowed us to abandon the two stages road axis design - the map projection and the cross projection - in favor of defining the road axis as a spatial curve in the form of parametric splines, the smoothness of connection of curves and surfaces comprising the roadway remains an open question. The authors further develop the cyclographic method in road surface formation and study the problem of smoothness of connection of ruled surfaces segments generated through the cyclographic mapping of a spatial curve. The present paper considers the aspects of smooth connection of polynomial spline curve segments and the respective cyclographic projections, as well as ruled surface segments that are directed by these curves. The results of the study allow one to pre-define the desirable order of smoothness of the connected curve segments and ruled surface segments comprising the road surface forms on the stage of road axis design and subsequent road surface formation. This fact can serve as the basis for development of CAD systems for road surface forms of general and special purpose.
\end{abstract}

\section{Keywords}

Cyclographic method, mapping, geometric modeling, roads, road surface forms, smoothness of connection, ruled surface.

\section{Introduction}

The modern automated road design has demand for development of the design methods and the mathematical apparatus of the applied geometric model. The design method dominating in this area at the moment is the Delaunay triangulation method commonly applied in road design CAD systems $[1,2,3]$. There are, however, certain areas where the triangulation method does not provide the required geometric parameters of the resulting model of road surface form. Let us consider a vehicle as a particle point following a curvilinear trajectory $\bar{r}=\bar{r}(t)$. It is known that the first derivative of radius-vector $\bar{r}(t)$ with respect to parameter $t$ defines the velocity vector, while the second derivative defines the acceleration vector, and the third derivative defines the jerk vector [4,5]. Obviously, frequent and rapid changes in acceleration are frequent and rapid jerks that can potentially damage cargo, injure passengers and drivers. It is therefore essential to assure smoothness of jerk variation function as well as its continuity. This requires moving trajectory continuity of up to the fourth derivative of its vector function [4]. Specific areas of road design demand geometric models featuring segments of curves and surfaces generating road surface forms to have high orders of smoothness, such as, for example, highway design, virtual road surface formation models for testing self-driving capabilities of artificial intelligence, etc. [6]

There is a sufficient number of scientific publications on the topic of smoothness of connection of spatial curve segments defining the road axis. However, assuring smoothness of connection of surface segments forming the road surface remains an open question. At the moment there is a relatively low number of Russian studies dedicated to development of new geometric models of road surface forms

GraphiCon 2021: 31st International Conference on Computer Graphics and Vision, September 27-30, 2021, Nizhny Novgorod, Russia EMAIL: Lubchinov.E.V@yandex.ru (E. Lyubchinov); panchuk_k1@mail.ru (K. Panchuk)

ORCID: 0000-0003-2499-4866 (E. Lyubchinov); 0000-0001-9302-8560 (K. Panchuk)

(c) (i) (C) 2021 Copyright for this paper by its authors.

Use permitted under Creative Commons License Attribution 4.0 International (CC BY 4.0).

CEUR Workshop Proceedings (CEUR-WS.org) 
with specified geometric parameters. One of such studies worth mentioning is the mathematical model proposed by Professor Salkov N. A. [7]. It constitutes a system of equations describing road surface form as a ruled non-developable surface. Another noteworthy model was proposed by Professor Mufteev V. G. [4,8]. It is based on high-quality spline curves.

The authors of the present paper have proposed a geometric model of road surface formation through the method of cyclographic mapping $[9,10]$. As with other models of road surface formation, the core of this model is a spatial curve modeling road axis. This curve along with its cyclographic projection serve as directrices for ruled surfaces that form the carriage way, the road shoulder and the slopes. The proposed geometric model allows one to acquire an analytical solution to the problem of mathematical description of the formed ruled surfaces. However, the question of smoothness of connection of formed ruled surface segments remains open and requires dedicated studying.

It is therefore the objective of the present paper to study smooth connection of segments of ruled surfaces forming road surface forms. The starting point of the study is the correlation between smoothness of connection of surfaces segments and smoothness of connection of segments of the corresponding directrices. Here each pair of directing segments of a single ruled surface segment are bijectively correspondent in cyclographic mapping as a prototype and its cyclographic image.

\section{Aspects of connection of road surfaces and curves formed through the method of cyclographic mapping}

The studies of capabilities of the method of cyclographic mapping in road surface formation conducted by the authors yielded two geometric models [9]. The first of these models is based on the classic cyclographic projection of a spatial curve and allows one to acquire road surface forms in the form of developable surfaces. The analysis of the existing body of construction literature $[1,2,7]$ and the typical solutions applied in road surface design showed that a different model is required. This model is derived from the first one by means of a specific transformation and allows one to acquire road surface forms in the form of non-developable surfaces.

The classic cyclographic representation features half-angle at the mapping cone vertex between its axis and its generatrix $\alpha=45^{\circ}$ [11]. Obviously, this value of half-angle $\alpha$ does not result in road surface forms that comply with the current standards and road design rules. The authors have acquired the equations for cyclographic mapping of a spatial curve that allow for constant half-angle value within limits $\left(0^{\circ}, 90^{\circ}\right)$ as well as variable half-angle as a function of a certain parameter $t[9,12]$. Such cyclographic projections were called $\beta$ - and $\beta(t)$-projections respectively. These projections made the basis of the first and the second geometric models of road surface formation [9].

Let us consider the way road surface formation is performed. The road axis is given in the form of a spatial curve $\bar{P}(t)=(x(t), y(t), z(t))$ of smoothness $C^{k}, \quad t \in \boldsymbol{R}: T_{0} \leq t \leq T, k=1,2, \ldots$. A cyclographic projection $\bar{P}_{\beta(1,2)}(t)$ of the spatial curve $\bar{P}(t)$ is constructed [9,10]. The curves $\bar{P}(t)$ and $\bar{P}_{\beta(1,2)}(t)$ combined generate a ruled surface $\Phi$ (Figure 1). In order to achieve the desirable width, this surface is then trimmed by means of vertical cylindrical surfaces constructed through curves $\bar{P}_{e(1,2)}(t)$ equidistant with respect to the orthogonal projection $\bar{P}_{1}(t)$ of road axis $\bar{P}(t)$. This way the projecting cylindrical surfaces constructed through the curves $\bar{P}_{e(1,2)}(t)$ upon intersection with the ruled surface $\Phi$ form the sought spatial curves of carriage way edges $\bar{m}_{(1,2)}(t)$ (Figure1). Obviously, the road axis $\bar{P}(t)$ and the acquired edges $\bar{m}_{(1,2)}(t)$ constitute road surface form directrices. It should be noted that the cyclographic projection $\bar{P}_{\beta(1,2)}(t)$ in general consists of two branches. Figure 1 illustrates construction of carriage way edge $\bar{m}_{(1)}(t)$ of one of the two branches of the cyclographic projection, namely $\bar{P}_{\beta(1)}(t)$. 


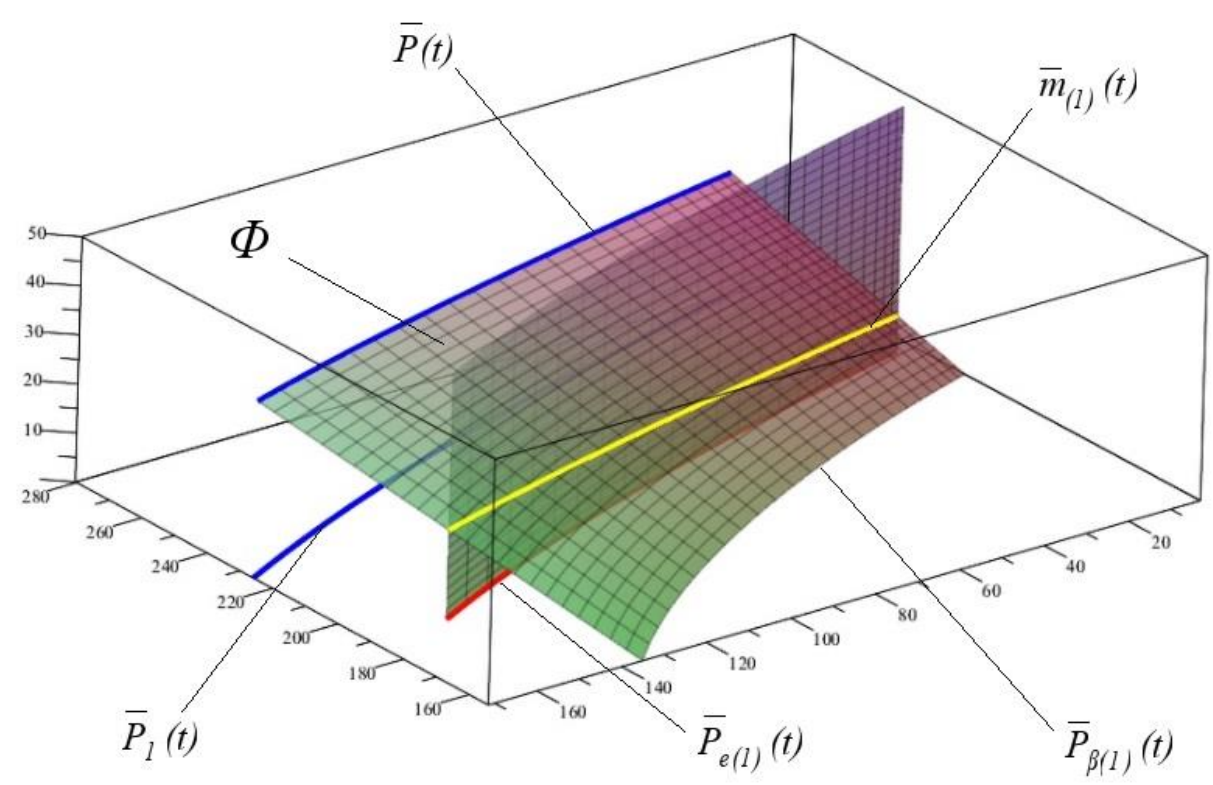

Figure 1: Carriage way edge $\bar{m}_{(1)}(t)$ construction scheme

\subsection{Aspects of connection of cyclographic projection segments}

The initial object in road surface formation is the trajectory that is the road axis. Every subsequent construction relies on the road axis. In most cases the road axis is given in the form of a spline curve consisting of a number of segments connected with a certain order of smoothness $[1,4,8,13,14,15]$. In the previous studies of the cyclographic approach in road surface formation the authors have established the correspondence between the smoothness of connection of the initial spline segments (road axis segments) and the respective cyclographic projection segments [16]. In addition, the following statement has been proven: subsequent fulfillment of equations of continuous derivatives of polynomial vector functions describing segments of order up to $k$ inclusive in the points of connection of spline curve segments $\bar{P}(t)=\sum_{i=0}^{k+1} \bar{A}_{i} \cdot t^{i}, t \in\left[T_{0}, T\right] \in \boldsymbol{R}^{1}$ is sufficient in order to achieve smoothness $C_{\beta}^{k-1}$ in the points of connection of the respective cyclographic images.

The above statement is true for a spatial polynomial spline curve with fixed boundary conditions. As follows from this statement, meeting certain conditions guarantees smoothness $C_{\beta}^{k-1}$ of connection of cyclographic projections. But is it at all possible to achieve smoothness $C_{\beta}^{k}$ of connection of cyclographic projections given the same initial conditions (smoothness $C_{\beta}^{k}$ of connection of the initial curve segments)? Paper [16] proves another statement: subsequent fulfillment of equations of continuous derivatives of polynomial vector functions describing segments of order up to $k+1$ inclusive in the points of connection of spline curve segments $\bar{P}(t)=\sum_{i=0}^{k+1} \bar{A}_{i} \cdot t^{i}, t \in\left[T_{0}, T\right] \in \boldsymbol{R}^{1}$ is sufficient in order to achieve smoothness $C_{\beta}^{k}$ in the points of connection of the respective cyclographic images. This statement is true in the case of a spatial polynomial spline curve with free boundary conditions.

Development of effective algorithms of cyclographic formation of composite curves and surfaces applied in road surface form design requires one to consider the correspondence between the smoothness of connection of the initial polynomial spline curve segments and smoothness of connection of the respective cyclographic projection segments.

\subsection{Connection smoothness of ruled surfaces segments}


Let us consider smoothness of connection of segments of a ruled surface formed by directing curves $\bar{P}(t)$ and $\bar{P}_{\beta}(t)$, where $\bar{P}(t)=\sum_{i=0}^{k+1} \bar{A}_{i} \cdot t^{i}, t \in\left[T_{0}, T\right] \in \boldsymbol{R}^{1}$ represents a polynomial spline segment, $\bar{P}_{\beta}(t)$ represents a cyclographic $\beta$-projection of this segment. The ruled surface $\Phi(t, l)$ under consideration is defined by the following equations:

$$
\begin{aligned}
& X(t, l)=x(t) \cdot(1-l)+l \cdot x_{\beta}(t), \\
& Y(t, l)=y(t) \cdot(1-l)+l \cdot y_{\beta}(t), \\
& Z(t, l)=z(t) \cdot(1-l), l \in\left[L_{0}, L\right] \in \boldsymbol{R}^{1},
\end{aligned}
$$

where $\bar{P}(t)=(x(t), y(t), z(t)), \overline{P_{\beta}}(t)=\left(x_{\beta}(t), y_{\beta}(t), 0\right)$.

A segment of the studied ruled surface $\Phi$ is depicted on figure 2. Let us express the first partial derivatives from the equations (1):

$$
\begin{aligned}
& X_{t}^{\prime}(t, l)=x_{t}^{\prime}(t) \cdot(1-l)+l \cdot\left(x_{\beta}\right)_{t}^{\prime}(t), \\
& Y_{t}^{\prime}(t, l)=y_{t}^{\prime}(t) \cdot(1-l)+l \cdot\left(y_{\beta}\right)_{t}^{\prime}(t), \\
& Z_{t}^{\prime}(t, l)=z_{t}^{\prime}(t) \cdot(1-l) . \\
& X_{l}^{\prime}(t, l)=x_{\beta}(t)-x(t), \\
& Y_{l}^{\prime}(t, l)=y_{\beta}(t)-y(t), \\
& Z_{l}^{\prime}(t, l)=-z(t) .
\end{aligned}
$$

As follows from the definition of a smooth $C^{k}$ surface $(k \in N)$ [17], there have to be continuous partial derivatives of orders up to $k$ inclusive of coordinate functions $X(t, l), Y(t, l)$, and $Z(t, l)$ in every point of such surface. The following condition also has to be true:

$$
\operatorname{rank}\left(\begin{array}{lll}
X_{t}^{\prime}(t, l) & Y_{t}^{\prime}(t, l) & Z_{t}^{\prime}(t, l) \\
X_{l}^{\prime}(t, l) & Y_{l}^{\prime}(t, l) & Z_{l}^{\prime}(t, l)
\end{array}\right)=2 .
$$

Obviously, the geometric condition (4) indicates linear independence of vector derivatives $\bar{P}_{t}^{\prime}$ and $\bar{P}_{l}^{\prime}$, i.e. existence of a non-zero normal vector $\bar{N}=\left[\bar{P}_{t}^{\prime}, \bar{P}_{l}^{\prime}\right]$ at every point of the surface. Since $\bar{P}(t)$ is a polynomial spline segment, the condition (4) is fulfilled. It is also obvious that partial derivatives $x_{t}^{(k+1)}(t), y_{t}^{(k+1)}(t), z_{t}^{(k+1)}(t)$ become constant, therefore the following holds true for derivatives of $\operatorname{order}(k+j)$ :

$$
X_{t}^{(k+j)}(t, l)=l \cdot\left(x_{\beta}\right)_{t}^{(k+j)}(t) ; Y_{t}^{(k+j)}(t, l)=l \cdot\left(y_{\beta}\right)_{t}^{(k+j)}(t) ; Z_{t}^{(k+j)}(t, l)=0, j=2,3,4, \ldots .
$$

It is once again obvious that every partial derivative acquired subsequently through equations (3) starting with the second order is equal to zero:

$$
X_{l}^{(j)}(t, l)=Y_{l}^{(j)}(t, l)=Z_{l}^{(j)}(t, l)=0, \quad j=2,3,4, \ldots .
$$

Therefore, the right parts of the equations (1) have continuous partial derivatives of every order; in addition, in every point $(t, l) \in \boldsymbol{R}^{2}$ of surface $\Phi$ the following condition is true:

$$
\operatorname{rank}\left(\begin{array}{lll}
X_{t}^{\prime}(t, l) & Y_{t}^{\prime}(t, l) & Z_{t}^{\prime}(t, l) \\
X_{l}^{\prime}(t, l) & Y_{l}^{\prime}(t, l) & Z_{l}^{\prime}(t, l)
\end{array}\right)=2 .
$$

For this reason a segment of ruled surface $\Phi$ constructed on the basis of a polynomial spline segment and its cyclographic projection has smoothness $C^{\infty}$. 


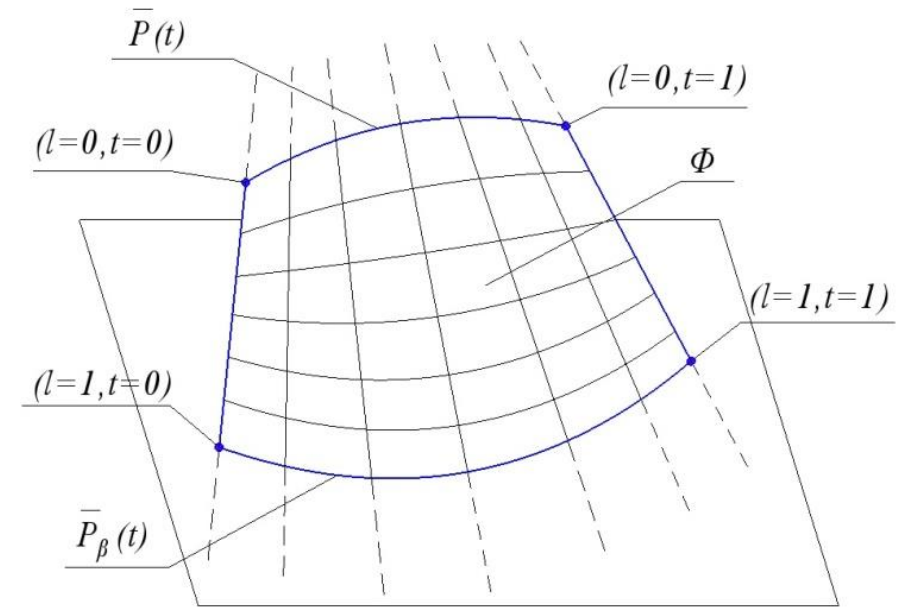

Figure 2: A segment of ruled surface $\Phi$

Connection of polynomial spline segments $\bar{P}(t)=\sum_{i=0}^{k+1} \bar{A}_{i} \cdot t^{i}, t \in \boldsymbol{R}^{1}$ with continuity $C^{k}$ at the point of connection that forms a composite spline curve and subsequent construction of a cyclographic projection of said curve allows one to construct a composite ruled surface $\Phi$. Segments of this surface are formed by the segments of directrices $\bar{P}(t)$ and $\bar{P}_{\beta}(t)$. Smoothness of connection of ruled segments at the common generatrix depends on smoothness of connection of the initial spline curve segments. Let us express the equations for partial derivatives of order $k$ from the equations (1):

$$
\begin{aligned}
& X_{t}^{(k)}(t, l)=x_{t}^{(k)}(t) \cdot(1-l)+l \cdot\left(x_{\beta}\right)_{t}^{(k)}(t), \\
& Y_{t}^{(k)}(t, l)=y_{t}^{(k)}(t) \cdot(1-l)+l \cdot\left(y_{\beta}\right)_{t}^{(k)}(t), \\
& Z_{t}^{(k)}(t, l)=z_{t}^{(k)}(t) \cdot(1-l) . \\
& X_{l}^{(k)}(t, l)=Y_{l}^{(k)}(t, l)=Z_{l}^{(k)}(t, l)=0, k \geq 2 .
\end{aligned}
$$

Figure 3 depicts the connected ruled segments $\bar{r}_{n}=\bar{r}_{n}(t, l)$ and $\bar{r}_{n+1}=\bar{r}_{n+1}(t, l)$ with pairs of directrices $\bar{P}_{n}, \bar{P}_{\beta(n)}$ and $\bar{P}_{n+1}, \bar{P}_{\beta(n+1)}$, where $\bar{P}_{n}(t)=\sum_{i=0}^{k+1} \bar{A}_{i}^{(n)} \cdot t^{i}, \bar{P}_{n+1}(t)=\sum_{i=0}^{k+1} \bar{A}_{i}^{(n+1)} \cdot t^{i}$. Segments $\bar{P}_{\beta(n)}$ and $\bar{P}_{\beta(n+1)}$ are acquired through the equations for cyclographic projection of a spatial curve [9,11]. Connection of segments $\bar{P}_{n}$ and $\bar{P}_{n+1}$ with smoothness $C^{k}$ requires the following conditions at the point of connection:

$$
\bar{P}_{n}^{\prime}(t=1)=\bar{P}_{n+1}^{\prime}(t=0), \ldots, \bar{P}_{n}^{(k)}(t=1)=\bar{P}_{n+1}^{(k)}(t=0) .
$$

Here smoothness $C^{k}$ corresponds to smoothness $C_{\beta}^{k-1}$ the same way as smoothness $C^{k+1}$ corresponds to smoothness $C_{\beta}^{k}$ depending on the boundary conditions set for spline curve $\bar{P}_{n} \cup \bar{P}_{n+1}$ formation (see subsection 2.1). Based on the equations (5), (6), (7) and the respective geometric representation, as well as the above definition of ruled surface segment smoothness, the following conclusion can be drawn: partial derivatives $\left(\bar{r}_{n}\right)_{t}^{(k-1)}$ and $\left(\bar{r}_{n+1}\right)_{t}^{(k-1)}$ of orders up to $(k-1)$ inclusive of equations $\bar{r}_{n}=\bar{r}_{n}(t, l)$ and $\bar{r}_{n+1}=\bar{r}_{n+1}(t, l)$ for ruled surface segments constructed of polynomial splines and smoothly connected at the common generatrix $l_{n, n+1}$ are equal in the points of this generatrix if the derivatives of orders up to $k$ inclusive of coordinate functions of polynomial spline segments $\bar{P}_{n}(t)$ and $\bar{P}_{n+1}(t)$ are equal:

$$
x_{n}^{(k)}(t=1)=x_{n+1}^{(k)}(t=0), y_{n}^{(k)}(t=1)=y_{n+1}^{(k)}(t=0), z_{n}^{(k)}(t=1)=z_{n+1}^{(k)}(t=0) .
$$

Indeed, as follows from equation (8),

$$
\bar{P}_{\beta(n)}(t=1)=\bar{P}_{\beta(n+1)}(t=0), \bar{P}_{\beta(n)}^{\prime}(t=1)=\bar{P}_{\beta(n+1)}^{\prime}(t=0), \ldots, \bar{P}_{\beta(n)}^{(k-1)}(t=1)=\bar{P}_{\beta(n+1)}^{(k-1)}(t=0),
$$

which allows us to express the following with consideration for the equations (6): 


$$
\left(\bar{r}_{n}\right)_{t}^{(k-1)}=\left(\bar{r}_{n+1}\right)_{t}^{(k-1)} .
$$

Furthermore, as follows from equations (7), $\left(\bar{r}_{n}\right)_{l}^{(k-1)}=\left(\bar{r}_{n+1}\right)_{l}^{(k-1)}=0$.

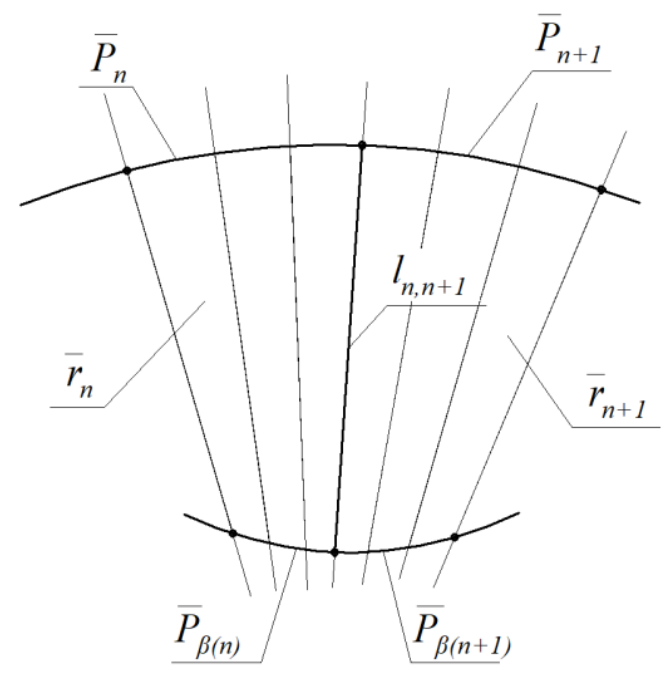

Figure 3: Connection of ruled surface segments

As one can conclude from the above, the smoothness $C^{k-1}$ of connection of ruled surface segments directed by a spatial spline curve $\bar{P}$ and the respective cyclographic projection $\bar{P}_{\beta}$ at the common generatrix corresponds to the smoothness $C_{\beta}^{k-1}$ of connection of cyclographic projection segments given fixed boundary conditions of the initial polynomial spline curve. If the boundary conditions are free, ruled surface segments are connected with smoothness $C_{\beta}^{k}$. Obviously, equations (11) can serve as the basis for formation of a smooth composite ruled surface applicable as the carriage way of a road.

\section{Results of experiments}

Let us consider an example. Consider a polynomial spline curve $\bar{P}(t)$ consisting of third-degree Bezier curve segments given in the following form:

$$
\bar{P}(t)=(1-t)^{3} A_{0}+3 t(1-t)^{2} A_{1}+3 t^{2}(1-t) A_{2}+t^{3} A_{3}, t \in[0,1] .
$$

Let the considered spline curve be passing through four points with the following coordinates (in millimeters): $Q_{0}=(0 ; 0 ; 4), Q_{1}=(10 ; 1 ; 3), Q_{2}=(20 ;-1 ; 4), Q_{3}=(30 ; 1 ; 5)$. Let us also accept boundary conditions: second derivatives in boundary points are equal to zero. Then the equations of the three polynomial spline curve segments $\bar{P}_{01}(t), \bar{P}_{12}(t), \bar{P}_{23}(t)$ with the above conditions are of the following form:

$$
\begin{aligned}
& x_{01}=10 t(1-t)^{2}+20 t^{2}(1-t)+10 t^{3} ; \\
& y_{01}=2,07 t(1-t)^{2}+4,13 t^{2}(1-t)+t^{3} ; \\
& z_{01}=4(1-t)^{3}+10,5 t(1-t)^{2}+8,93 t^{2}(1-t)+3 t^{3} ; \\
& x_{12}=10(1-t)^{3}+40 t(1-t)^{2}+50 t^{2}(1-t)+20 t^{3} ; \\
& y_{12}=(1-t)^{3}+1,87 t(1-t)^{2}-2,47 t^{2}(1-t)-t^{3} ; \\
& z_{12}=3(1-t)^{3}+9,07 t(1-t)^{2}+10,73 t^{2}(1-t)+4 t^{3} ; \\
& x_{23}=20(1-t)^{3}+69,9 t(1-t)^{2}+80 t^{2}(1-t)+30 t^{3} ; \\
& y_{23}=-(1-t)^{3}-1,53 t(1-t)^{2}-0,26 t^{2}(1-t)+t^{3} ; \\
& z_{23}=4(1-t)^{3}+13,27 t(1-t)^{2}+14,13 t^{2}(1-t)+5 t^{3} ;
\end{aligned}
$$


where $0 \leq t \leq 1$.

Let us substitute the coordinate functions of vector equations for segments $\bar{P}_{01}(t), \bar{P}_{12}(t), \bar{P}_{23}(t)$ into the equation for the cyclographic $\beta$-projection (half-angle $\beta$ at the projecting cone vertex is equal to $1 \mathrm{rad})$ of the following form $[9,11,12]$ :

$$
\begin{aligned}
& x_{\beta(1,2)}(t)=x(t)+z(t) \cdot e \frac{-x(t)^{\prime} \cdot z(t)^{\prime} \cdot e \mp y(t)^{\prime} \sqrt{\left(x(t)^{\prime}\right)^{2}+\left(y(t)^{\prime}\right)^{2}-\left(z(t)^{\prime}\right)^{2} \cdot e^{2}}}{\left(x(t)^{\prime}\right)^{2}+\left(y(t)^{\prime}\right)^{2}}, \\
& y_{\beta(1,2)}(t)=y(t)+z(t) \cdot e \frac{-y(t)^{\prime} \cdot z(t)^{\prime} \cdot e \pm x(t)^{\prime} \sqrt{\left(x(t)^{\prime}\right)^{2}+\left(y(t)^{\prime}\right)^{2}-\left(z(t)^{\prime}\right)^{2} \cdot e^{2}}}{\left(x(t)^{\prime}\right)^{2}+\left(y(t)^{\prime}\right)^{2}},
\end{aligned}
$$

where $e=\operatorname{tg} \beta$.

Let us express the acquired equations for cyclographic projection $\bar{P}_{\beta}(t)$ segments in the following fashion: $\bar{P}_{\beta(01)}(t)=\left(x_{\beta(01)}, y_{\beta(01)}, z_{\beta(01)}\right), \bar{P}_{\beta(12)}(t)=\left(x_{\beta(12)}, y_{\beta(12)}, z_{\beta(12)}\right), \bar{P}_{\beta(23)}(t)=\left(x_{\beta(23)}, y_{\beta(23)}, z_{\beta(23)}\right)$. As follows from the first statement presented in subsection 2.1 and considering that the smoothness $C^{k}$ has order $k=2$, we conclude that smoothness of connection of cyclographic projection segments in the current example equals $C_{\beta}^{1}$.

By substitution of the acquired coordinate functions for vector equations of the initial spline curve $\bar{P}(t)$ segments and its respective cyclographic projection $\bar{P}_{\beta}(t)$ into the equations (1) we acquire the parametric equation of the three linear surface $\Phi$ segments:

$$
\begin{aligned}
& X_{01}=\left(-148,8 l-1042,7 t+20,7 l t^{5}-5,2 \cdot 10^{-7} l t^{4}-79,34 l t^{3}+155,23 l t^{2}+57,03 l t-12,87 l \cdot N-\right. \\
& \left.-102,4 t^{5}+132,27 t^{3}+19,9 l t^{2} \cdot N+4,94 l t \cdot N+2,66 l t^{5} \cdot N-9,36 l t^{3} \cdot N-4,98 l t^{4} \cdot N\right) / M ; \\
& Y_{01}=\left(30,74 l+215,5 t-29,7 l t^{5}+49,67 l t^{4}+34,7 l t^{3}-79,69 l t^{2}-11,79 l t-62,3 l \cdot N+35,3 t^{5}-\right. \\
& \left.-10,9 t^{7}-138,56 t^{3}+1,56 \cdot 10^{-7} l t^{2} \cdot N+23,9 l t \cdot N-8,3 l t^{3} \cdot N+6,6 l t^{7}-1,67 \cdot 10^{-7} l t^{6}\right) / M ; \\
& Z_{01}=0,53 t^{3}-10^{-8} t^{2}-1,53 t+4-0,53 l t^{3}+10^{-8} l t^{2}+1,53 l t-4 l ;
\end{aligned}
$$

where $M=104,27+10,24 t^{4}-13,23 t^{2}, N=98,7+4 t^{4}-1,33 t^{2}+7,97 \cdot 10^{-8} t^{3}-7,6 \cdot 10^{-8} t$.

$$
\begin{aligned}
& X_{12}=\left(4,85 l-1157,9 t+32,3 l t^{5}-129,36 l t^{4}+119,87 l t^{3}-137,77 l t^{2}+232,96 l t-29,22 l t^{2} \cdot N+\right. \\
& +30 l t \cdot N+7,27 l t^{5} \cdot N-24,09 l t^{4} \cdot N+14,36 l t^{3} \cdot N+406 t^{4}-t^{6}-490 t^{5}+4 \cdot 10^{-7} t^{7}+5,3 l \cdot N+ \\
& \left.+645,1 t^{3}-1,29 \cdot 10^{-7} l t^{7}+6,5 \cdot 10^{-7} l t^{6}-1012,84-396 t^{2}\right) / M ; \\
& Y_{12}=\left(-0,55 l+100,3 t+163 l t^{5}-158,5 l t^{4}+237,65 l t^{3}-130,1 l t^{2}-29,5 l t+24,9 l t^{2} \cdot N+1,04 l t \cdot N+\right. \\
& +4,15 \cdot 10^{-8} l t^{5} \cdot N-1,41 \cdot 10^{-7} l t^{4} \cdot N-10,38 l t^{3} \cdot N-104,1 t^{4}+365,9 t^{6}-289,7 t^{5}-114,3 t^{7}- \\
& \left.-101,3+46,7 l \cdot N-71,87 t^{3}+22,64 l t^{7}-111,25 l t^{6}+315,46 t^{2}\right) / M ; \\
& Z_{12}=-0,67 t^{3}+1,6 t^{2}+0,07 t+3-0,67 l t^{3}-1,6 l t^{2}-0,07 l t-4 l ;
\end{aligned}
$$

where $M=49 t^{4}-89,6 t^{3}+25,1 t^{2}+14,5 t+101,28, N=39,3 t^{4}-58,55 t^{3}+0,9 t^{2}+13,47 t+101,27$.

$$
\begin{aligned}
& X_{23}=\left(-8,35 l t^{2} \cdot N+46,29 l t \cdot N-0,79 l t^{5} \cdot N+3,95 l t^{4} \cdot N-12,34 l t^{3} \cdot N-122,89 l+840,7 t-\right. \\
& -1,29 l t^{5}+6,47 l t^{4}-24,15 l t^{3}-1,94 l t^{2}+38,7 l t-288,8 t^{4}+t^{6}+144,4 t^{5}-8 \cdot 10^{-7} t^{7}-537 t^{3}+ \\
& \left.+2005,69+2,59 \cdot 10^{-9} l t^{7}-1,55 \cdot 10^{-8} l t^{6}-3,32 l \cdot N+1155,2 t^{2}\right) / M ; \\
& Y_{23}=\left(6,23 l t^{2} \cdot N-19,7 l t \cdot N+4,15 \cdot 10^{-9} l t^{5} \cdot N-1,66 \cdot 10^{-8} l t^{4} \cdot N-2,08 l t^{3} \cdot N+6,55 l-45,4 t+\right. \\
& +14,16 l t^{5}-17,96 l t^{4}-14,89 l t^{3}+76,21 l t^{2}-95,46 l t+261,52 t^{4}+128,03 t^{6}-305,49 t^{5}-18,29 t^{7}- \\
& \left.-133,04 t^{7}-100,28+0,49 l t^{7}-3,44 l t^{6}+323,59 t^{2}-62,3 \cdot N\right) / M ; \\
& Z_{23}=0,13 t^{3}-0,4 t^{2}+1,27 t+4-0,13 l t^{3}+0,4 l t^{2}-1,27 l t-4 l ;
\end{aligned}
$$


where $M=14,44 t^{4}-57,76 t^{3}+61,81 t^{2}-8,1 t+100,28, N=14,05 t^{4}-56,21 t^{3}+57,8 t^{2}-3,19 t+96,4$.

Figure 4 illustrates the acquired segments of the surface $\Phi$. The visualization is performed in Maple computer algebra system.

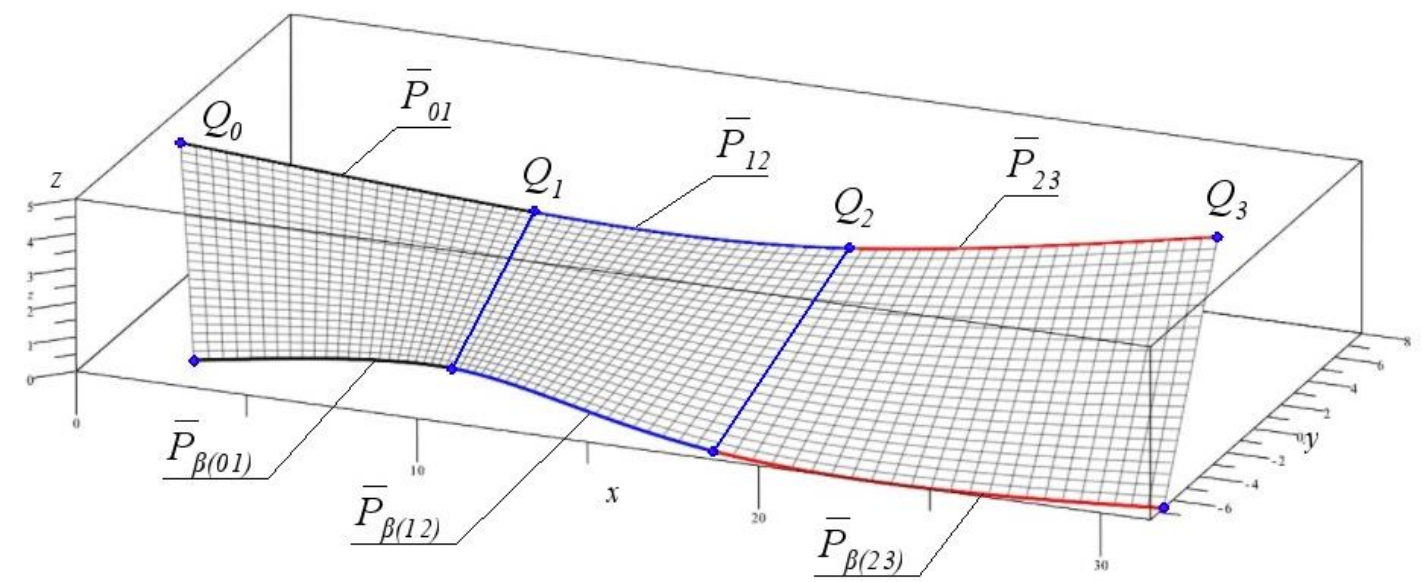

Figure 4: Visualization of the segments of the ruled surface $\Phi$

Starting from (11), let us find partial derivatives of the acquired equations for the segments of the surface $\Phi$ at the points of the common generatrix. The partial derivatives with respect to parameter $l$, as pointed out earlier, are equal to zero starting with the second one. In this regard, the partial derivatives with respect to parameter $t$ are presented below:

$$
\begin{gathered}
\left(X_{01}\right)^{\prime}(t=1)=\left(X_{12}\right)^{\prime}(t=0)=10-5,24 l ; \\
\left(Y_{01}\right)^{\prime}(t=1)=\left(Y_{12}\right)^{\prime}(t=0)=-1,13+0,54 l ; \\
\left(Z_{01}\right)^{\prime}(t=1)=\left(Z_{12}\right)^{\prime}(t=0)=0,67-0,67 l ; \\
\left(X_{12}\right)^{\prime}(t=1)=\left(X_{23}\right)^{\prime}(t=0)=10+4,8 l ; \\
\left(Y_{12}\right)^{\prime}(t=1)=\left(Y_{23}\right)^{\prime}(t=0)=-0,53-3,27 l ; \\
\left(Z_{12}\right)^{\prime}(t=1)=\left(Z_{23}\right)^{\prime}(t=0)=1,27-1,27 l ; \\
\left(X_{01}\right)^{\prime \prime}(t=1)=-4,58 l ;
\end{gathered}
$$

As we see from the presented results of partial derivatives calculation, the first partial derivatives with respect to parameter $t$ are equal, while the second partial derivatives are not. Therefore, the ruled surfaces segments are connected with smoothness $C^{1}$.

Let us now consider a case, where the initial spline curve $\bar{P}(t)$ has free boundary conditions. This allows us to specify an additional condition of equality of derivatives of order $(k+1)$ at the points of connection of its segments. As we know, the derivative of order $(k+1)$ of a polynomial spline $\bar{P}(t)=\sum_{i=0}^{k+1} \bar{A}_{i} \cdot t^{i}, t \in\left[T_{0}, T\right] \in \boldsymbol{R}^{1}$ is constant. In the considered case it is the third derivative. Then the equations of the initial segments $\bar{P}_{01}(t), \bar{P}_{12}(t), \bar{P}_{23}(t)$ are of the following form:

$$
\begin{aligned}
& x_{01}=10 t(1-t)^{2}+20 t^{2}(1-t)+10 t^{3} ; \\
& y_{01}=4,83 t(1-t)^{2}+4,67 t^{2}(1-t)+t^{3} ; \\
& z_{01}=4(1-t)^{3}+9,33 t(1-t)^{2}+8,67 t^{2}(1-t)+3 t^{3} ;
\end{aligned}
$$




$$
\begin{aligned}
& x_{12}=10(1-t)^{3}+40 t(1-t)^{2}+50 t^{2}(1-t)+20 t^{3} ; \\
& y_{12}=(1-t)^{3}+1,33 t(1-t)^{2}-1,83 t^{2}(1-t)-t^{3} ; \\
& z_{12}=3(1-t)^{3}+9,33 t(1-t)^{2}+10,67 t^{2}(1-t)+4 t^{3} ; \\
& x_{23}=20(1-t)^{3}+69,9 t(1-t)^{2}+80 t^{2}(1-t)+30 t^{3} ; \\
& y_{23}=-(1-t)^{3}-4,17 t(1-t)^{2}-3,33 t^{2}(1-t)+t^{3} ; \\
& z_{23}=4(1-t)^{3}+13,33 t(1-t)^{2}+14,67 t^{2}(1-t)+5 t^{3} ;
\end{aligned}
$$

where $0 \leq t \leq 1$.

Let us apply the algorithm above and acquire new equations for the ruled surface $\Phi$ segments. Let us check whether their second derivatives with respect to parameter $t$ at the points of the common generatrix are equal:

$$
\begin{aligned}
& \left(X_{01}\right)^{\prime \prime}(t=1)=\left(X_{12}\right)^{\prime \prime}(t=0)=3,83 l ; \\
& \left(Y_{01}\right)^{\prime \prime}(t=1)=\left(Y_{12}\right)^{\prime \prime}(t=0)=-3-2,33 l ; \\
& \left(Z_{01}\right)^{\prime \prime}(t=1)=\left(Z_{12}\right)^{\prime \prime}(t=0)=2-2 l ; \\
& \left(X_{12}\right)^{\prime \prime}(t=1)=\left(X_{23}\right)^{\prime \prime}(t=0)=-4 \cdot 10^{-8}+8,06 l ; \\
& \left(Y_{12}\right)^{\prime \prime}(t=1)=\left(Y_{23}\right)^{\prime \prime}(t=0)=4-1,73 l ; \\
& \left(Z_{12}\right)^{\prime \prime}(t=1)=\left(Z_{23}\right)^{\prime \prime}(t=0)=-1 \cdot 10^{-8}+1 \cdot 10^{-8} l .
\end{aligned}
$$

The equality of the second derivatives with respect to parameter $t$ along the common generatrix follows from the calculation results. This means that ruled surface segments are connected with smoothness $C^{2}$. It is possible to draw a conclusion that smoothness $C_{\beta}^{k}$ or $C_{\beta}^{k-1}$ of connection of cyclographic projection segments determines smoothness of connection of the constructed segments of ruled surfaces of respective degrees $k$ and $(k-1)$. This conclusion confirms the theoretical results acquired in subsection 2.2.

\section{Conclusion}

The paper considers the problem of smoothness of connection of segments of ruled surfaces acquired through cyclographic mapping of a spatial spline curve and applied in road surface form modeling. The results of theoretical studies and numerical experiments have shown that ruled surface segments directed by segments of a polynomial spline curve and its cyclographic projection are connected with order of smoothness equal to the order of smoothness of connection of the cyclographic projection segments. The results of the studies on smoothness of connection of ruled surface segments within the proposed geometric model can find application in formation of road surface forms of general and special purpose.

\section{References}

[1] V. N. Boykov, G. A. Fedotov, V. I. Purkin, Automated Automotive Road Design (on the example of IndorCAD/Road), MADI, Moscow, 2005 (in Russian).

[2] V. I. Purkin, Basic Automated Design of Automotive Roads, MADI, Moscow, 2000 (in Russian).

[3] I.V. Krivyh, N.S. Mirza, Overview of foreign CAD systems for highways. SAPR i GIS avtomobil'nyh dorog 2(5) (2015). doi: 10.17273/CADGIS.2015.2.11.

[4] V. G. Mufteev, V.V. Shabakeeva, I.N. Aslyamov, YU.I. Lukmanov, I.O. Evtyagina. Designing the optimal road alignment in Indorcad using the "FAIR CURVE MODELER" program, Information Technology. Problems and solutions 4(9) (2019).

[5] D. Eager, Ann-M. Pendrill, N. Reistad. Beyond velocity and acceleration: jerk, snap and higher derivatives. European Journal of Physics 37(6) (2016). doi: 10.1088/0143-0807/37/6/065008. 
[6] NVIDIA Drive Sim и Drive Constellation, 2021. URL: https://www.nvidia.com/ru-ru/selfdriving-cars/drive-constellation.

[7] N. A. Salkov. Modeling the geometric forms of highways, INFRA-M, Moscow, 2019 (in Russian).

[8] V.G. Mufteev, A.R. Mardanov. Geometric modeling of curved lines and high quality surfaces, Prikladnaya geometriya. Applied Geometry 18(8) (2006).

[9] K.L. Panchuk, A. S. Niteyskiy, E. V. Lyubchinov, Cyclographic Modeling of Surface Forms of Highways, IOP Conf. Series: Materials Science and Engineering 262, 2017. doi: 10.1088/1757899X/262/1/012108.

[10] K. L. Panchuk, E.V. Lyubchinov, T.M. Myasoedova, Cyclography. Aspects of Theory and Practical Applications, in: 28th The 28th International Conference on Computer Graphics and Vision. Conf. Proceedings, GraphiCon 2018, Tomsk, 2018.

[11] K. L. Panchuk, N. V. Kaygorodtseva, Cyclographic Desctiptive Geometry, OmGTU, Omsk, 2017.

[12] E.V. Lyubchinov, A. S. Niteyskiy, K.L. Panchuk, Geometric modeling of curved reflectors, «Young Russia: advanced technologies - into industry», 1 (2017).

[13] D. Salomon, Curves and surfaces for computer graphics, Springer Science+Business Media Inc, 2006.

[14] G. Farin, Curves and surfaces for Computer Aided Geometric Design, Academic Press Inc., San Diego (USA), 1997.

[15] M. K. Agoston, Computer Graphics and Geometric Modeling, Springer-Verlag London, 2005.

[16] K. L. Panchuk, E.V. Lyubchinov, On smoothness of connection of spatial curve cyclographic projection, Journal of Physics: Conference Series 1791, 2021. doi: 10.1088/17426596/1791/1/012056.

[17] L. S. Atanasyan, V. T. Bazylev, Geometry. Part 2, Prosveshchenie, Moscow, 1987 (in Russian). 